\title{
Test of the Pauli Exclusion Principle in the VIP-2 Underground Experiment ${ }^{\dagger}$
}

\author{
Catalina Curceanu $1,2,3, *, \neq, \S$, Hexi Shi ${ }^{1,4, *, \$}$, Sergio Bartalucci ${ }^{1}$, Sergio Bertolucci ${ }^{5}$, \\ Massimiliano Bazzi ${ }^{1}$, Carolina Berucci ${ }^{1,4}$, Mario Bragadireanu ${ }^{1,3}$, Michael Cargnelli 1,4,
} Alberto Clozza ${ }^{1}$, Luca De Paolis ${ }^{1}$, Sergio Di Matteo ${ }^{6}$, Jean-Pierre Egger ${ }^{7}$, Carlo Guaraldo ${ }^{1}$, Mihail Iliescu ${ }^{1}$, Johann Marton ${ }^{1,4}$, Matthias Laubenstein ${ }^{8}$, Edoardo Milotti ${ }^{9}$, Marco Miliucci ${ }^{1}$, Andreas Pichler ${ }^{1,4}$, Dorel Pietreanu ${ }^{1,3}$, Kristian Piscicchia ${ }^{2,1}$, Alessandro Scordo ${ }^{1}$, Diana Laura Sirghi ${ }^{1,3}$, Florin Sirghi ${ }^{1,3}$, Laura Sperandio ${ }^{1}$, Oton Vazquez Doce ${ }^{1,10}$, Eberhard Widmann ${ }^{4}$ and Johann Zmeskal ${ }^{1,4}$

1 Laboratori Nazionali di Frascati, INFN, I-00044 Frascati, Italy; Sergio.bartalucci@lnf.infn.it (S.B.); massimiliano.bazzi@lnf.infn.it (Mas.B.); Carolina.Berucci@oeaw.ac.at (C.B.); Bragadireanu.Mario@lnf.infn.it (Mar.B.); michael.cargnelli@oeaw.ac.at (M.C.); alberto.clozza@lnf.infn.it (A.C.); luca.depaolis@lnf.infn.it (L.D.P.); carlo.guaraldo@lnf.infn.it (C.G.); Mihai.Iliescu@lnf.infn.it (M.I.); johann.marton@oeaw.ac.at (J.M.); marco.miliucci@lnf.infn.it (M.M.); Andreas.Pichler@oeaw.ac.at (A.P.); dorel.pietreanu@lnf.infn.it (D.P.); kristian.piscicchia@gmail.com (K.P.); alessandro.scordo@lnf.infn.it (A.S.); Diana.Laura.Sirghi@lnf.infn.it (D.L.S.);

Sirghi.FlorinCatalin@lnf.infn.it (F.S.); laura.sperandio@lnf.infn.it (L.S.); oton.vazquez@universe-cluster.de (O.V.D.); johann.zmeskal@oeaw.ac.at (J.Z.)

2 CENTRO FERMI - Museo Storico della Fisica e Centro Studi e Ricerche 'Enrico Fermi', I-00184 Rome, Italy

3 Institutul National pentru Fizica si Inginerie Nucleara Horia Hulubbei, IFIN-HH, R-077125 Magurele, Romania

4 Stefan-Meyer-Institute for Subatomic Physics, Austrian Academy of Science, A-1090 Vienna, Austria; eberhard.widmann@oeaw.ac.at

5 Dipartimento di Fisica e Astronomia, Universitá di Bologna, I-40127 Bologna, Italy; Sergio.Bertolucci@Lnf.infn.it

6 Institut de Physique UMR CNRS-UR1 6251, Université de Rennes1, F-35042 Rennes, France; sergio.dimatteo@univ-rennes1.fr

7 Institut de Physique, Université de Neuchâtel, CH-2000 Neuenburg, Switzerland; jean-pierre.egger@net2000.ch

8 Laboratori Nazionali del Gran Sasso, INFN, I-67100 Assergi L'Aquila, Italy; Matthias.laubenstein@lngs.infn.it

9 Dipartimento di Fisica, Universitá di Trieste and INFN-Sezione di Trieste, I-34127 Trieste, Italy; edoardo.milotti@ts.infn.it

10 Excellence Cluster Universe, Technische Universität München, D-85748 Garching, Germany

* Correspondence: Catalina.Curceanu@lnf.infn.it (C.C.); hexi.shi@lnf.infn.it (H.S.); Tel.: +39-06-9403-2321 (C.C.)

+ This paper is an extended version of our paper published in the XIV International Conference on Topics in Astroparticle and Underground Physics (TAUP2015), 7-11 September 2015, Torino, Italy.

$\ddagger$ Current address: Laboratori Nazionali di Frascati, INFN, Via E. Fermi 40, I-00044, Frascati, Italy.

$\S$ These authors contributed equally to this work.

Received: 29 April 2017; Accepted: 22 June 2017; Published: 24 June 2017

Abstract: The validity of the Pauli exclusion principle-a building block of Quantum Mechanics-is tested for electrons. The VIP (violation of Pauli exclusion principle) and its follow-up VIP-2 experiments at the Laboratori Nazionali del Gran Sasso search for X-rays from copper atomic transitions that are prohibited by the Pauli exclusion principle. The candidate events-if they exist—originate from the transition of a $2 p$ orbit electron to the ground state which is already occupied by two electrons. The present limit on the probability for Pauli exclusion principle violation for electrons set by the VIP experiment is $4.7 \times 10^{-29}$. We report a first result from the VIP-2 experiment 
improving on the VIP limit, which solidifies the final goal of achieving a two orders of magnitude gain in the long run.

Keywords: Pauli exclusion principle; quantum foundations; X-ray spectroscopy; underground experiment; silicon drift detector

\section{Introduction}

The Pauli exclusion principle (PEP) states that in a system there cannot be two (or more) fermions with all quantum numbers identical, and is a fundamental principle in physics. The validity of the PEP is the basis of the periodic table of elements, electric conductivity in metals, the degeneracy pressure which makes white dwarfs and neutron stars stable, as well as many other phenomena in physics, chemistry, and biology. In quantum mechanics $(\mathrm{QM})$, the states of particles are described in terms of wave functions. For identical particles, with respect to their permutation, the states are necessarily either symmetric for bosons, or antisymmetric for fermions. This "symmetrization postulate" [1] excludes the mixing of different symmetrization groups, and it is at the basis of the PEP. Messiah and Greenberg noted in [2] that this superselection rule "does not appear as a necessary feature of the QM description of nature". In this context, the violation of PEP is equivalent to the violation of spin-statistics [3], and experimentally to the existence of states of particles that follow statistics other than the fermionic or the bosonic ones.

Exhaustive reviews of the experimental and theoretical searches for a small violation of the PEP or the violation of spin-statistics can be found, for example, in [3,4]. We first point out that there is no established model in quantum field theory that can explicitly include small violations of the PEP. Secondly, although many experimental searches present limits for the violation, the parameters that quantify the limits are model/system-dependent and are not generally comparable. Moreover, in order to search for states that are in a mixed symmetry, it is crucial to introduce new states into the system, among which the PEP-violating states may be found. Ramberg and Snow [5] took this argument into account by running a high electric DC current through a copper conductor, and they searched for X-rays from transitions that are PEP-forbidden after electrons are captured by copper atoms. In particular, they searched for PEP-violating transitions from the $2 p$ level to the $1 s$ level, which is already occupied by two electrons. Due to the shielding effect of the additional electron in the ground level, the energy of such abnormal transitions will deviate from the copper $\mathrm{K} \alpha \mathrm{X}$-ray at $8 \mathrm{keV}$ by about $300 \mathrm{eV}$ [6], which are distinguishable in precision spectroscopic measurements. Since the new electrons from the current are supposed to have no a-priori established symmetry with the electrons inside the copper atoms, the detection of the energy-shifted X-rays is an explicit indication of the violation of spin-statistics, and thus the violation of the PEP for electrons.

We want to mention that one known system in which the dichotomy of fermions and bosons does not work is in the two-dimensional condensed matter physics through the (fractional) quantum Hall effect [7]. Particles that are neither fermions nor bosons, and that may exist in electronic systems confined to two spatial dimensions have been constructed theoretically and investigated in the laboratory with great consistency with the theories as reviewed in [8]. The physics of this special system is exciting in itself and may provide hints to the searches for the violation of the PEP in other systems.

In Section 2, we will introduce the VIP (violation of Pauli exclusion principle) and VIP-2 experiments at Laboratori Nazionali del Gran Sasso (LNGS), and in Section 3 the first results from the physics run of VIP-2 in 2016, which already improved the best result previously achieved by the VIP experiment with 3 years of data collection. The paper ends with conclusions and future perspectives. 


\section{VIP-2 Experiment}

The first experiment performed in the LNGS-INFN underground laboratory-the VIP experiment-used a similar method as that of Ramberg and Snow, and the same definition of the parameter to represent the probability that the PEP is violated, for a direct comparison of the experimental results. An improvement in sensitivity was achieved firstly by performing the experiment in the low radioactivity laboratory at LNGS, which has the advantage of the excellent shielding against cosmic rays. Secondly, the application of charge-coupled device (CCD) as the X-ray detector with a typical energy resolution of $320 \mathrm{eV}$ at $8 \mathrm{keV}$ increased the precision in the definition of the region of interest to search for anomalous $X$-rays. The VIP experiment set the limit for the probability of the PEP violation for electrons to be $4.7 \times 10^{-29}$ [9-11].

By using new $\mathrm{X}$-ray detectors and an active shielding of scintillators, the VIP-2 experiment plans to further improve the sensitivity by two orders of magnitude. The major improvements come from the change of the layout of the copper strip target and of the X-ray detectors, which allow a larger acceptance for the X-ray detection. Secondly, a DC current with 100 amperes is applied instead of 40 amperes, which introduces two times the new electrons into the copper strip. Finally, in addition to the improved passive shielding surrounding the setup to reduce the background generated by the environmental radiations, the use of silicon drift detectors (SDDs) as the X-ray detectors allows the implementation of an active shielding using scintillators, as illustrated in Figure 1a, which removes the background induced by the high-energy charged particles that are not shielded. More details of the detectors and the VIP-2 setup are given in [12-15].

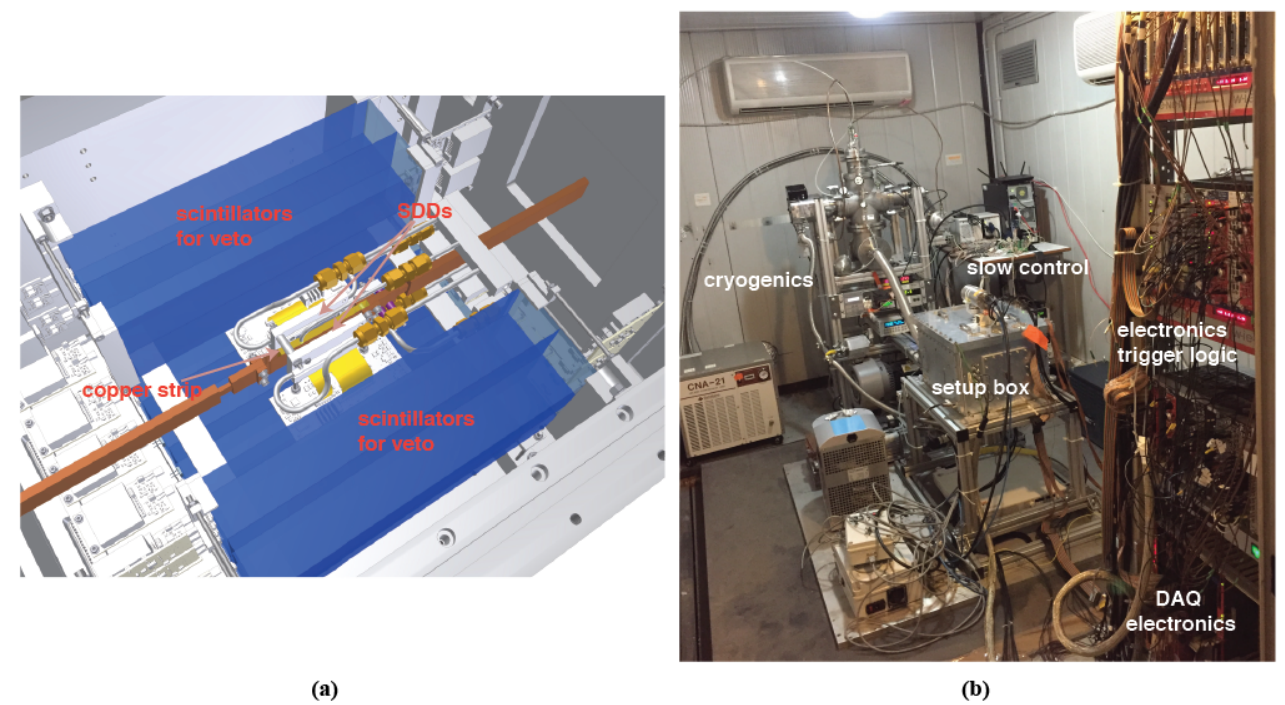

Figure 1. (a) The design of the core components of the VIolation of Pauli exclusion principle 2 (VIP-2) setup, including the silicon drift detectors (SDDs) as the X-ray detector, the scintillators as active shielding with silicon photomultiplier readout; (b) a picture of the VIP-2 setup in operation at the underground laboratory of Gran Sasso.

The VIP-2 trigger logic was implemented using the Nuclear Instrumentation Module (NIM) standard modules, and it is defined by either an event at any SDD or a coincidence between two layers of the veto detector. A Versa Module Europa (VME) based data acquisition system for the detectors was constructed. It records the energy deposit of the six SDDs from the output of a CAEN 568 spectroscopy amplifier which processes the analog signals of the SDD preamplifier output. The charge to digital signals (QDC) of the 32 scintillator channels, and the timing information of the SDDs with respect to the main trigger are recorded in the data as well. The data acquisition computer transfers data from the VME whenever there is one event ready in the memories of the modules, and clears the registers of the VME when the data transfer is done. During the whole communication process between the 
computer and the VME controller, the trigger logic is prohibited from receiving further events. The user interface of the Labview-based data-taking program can be remotely accessed and controlled from the computer terminals outside the Gran Sasso laboratory.

The temperatures of the SDDs, the copper conductor, the cooling system, as well as the ambient temperature and vacuum pressure of the setup are monitored by a slow control system. The slow control which can be accessed from remote terminals also controls the DC power supply to switch on and off the current applied to the copper strip. A closed circuit chiller coupled to a cooling pad attached to the copper strips keeps a constant temperature below 25 Celsius of the strips when the DC current up to $100 \mathrm{~A}$ is applied. The temperature of the SDDs' holder frame had a change of less than 2 $\mathrm{K}$ when the $100 \mathrm{~A}$ current was applied to the copper strip. At this level of temperature variation, the effect of change in the energy resolution of the SDDs is negligible.

In November 2015, after having performed exhaustive tests in the laboratory, the VIP-2 setup was transported and mounted in the Gran Sasso underground laboratory, as shown in Figure $1 \mathrm{~b}$. After tuning and optimization, from October 2016 we started the first campaign of data taking with the complete detector system. The energy calibration of the SDDs was performed in in-situ, by placing a weak Iron- 55 source covered by a $25 \mu \mathrm{m}$-thick titanium foil near the detectors. The manganese $K$-series $\mathrm{X}$-rays from the source partly go through the foil and partly irradiate the foil, generating titanium $\mathrm{K}$-series X-rays. These fluorescence X-rays are detected by the SDDs at an overall rate of about $2 \mathrm{~Hz}$, and provide reference energy peaks to calibrate the digitized SDD signals to energy scale.

\section{First VIP-2 Results}

During the data collection from October to December 2016, the DC current was typically switched on for one week and off for the next. The energy calibrations for the SDDs were done for each data set corresponding to a period of about one week, and then summed separately over the whole data collection period of over two months, for $100 \mathrm{~A}$ current-on data and current-off data sets. The spectra that correspond to 34 days of effective data acquisition with $100 \mathrm{~A}$ current on and 28 days with current off are shown in Figure 2, in which the fluorescence lines of titanium and manganese are marked.

The environmental gamma radiations and high-energy charged particles can irradiate the copper conductor or the strip inside the setup, and the normal $K$-series $\mathrm{X}$-rays from the de-excitation of the copper form the main background near the energy region of interest (ROI in Figure 2) from $7629 \mathrm{eV}$ to $7829 \mathrm{eV}$, which is defined by the SDD energy resolution ( $200 \mathrm{eV}$ full width at half maximum, FWHM) at the $K_{\alpha}$ copper transition $(8.04 \mathrm{keV})$ near the expected value of the PEP violating transition. In order to obtain the number of events violating PEP in the ROI, the current-on spectrum was normalized to 28 days of data collection time, and then a subtraction with the current-off spectrum was performed. The numbers of $\mathrm{X}$-rays in the region of interest were :

- $\quad$ with $\mathrm{I}=100 \mathrm{~A} ; N_{X}=2222 \pm 47$ (for 34 days of data collection);

- $\quad$ with $\mathrm{I}=0 \mathrm{~A} ; N_{X}=2181 \pm 47$ (28 days of data collection normalized to 34 days);

- numerical subtraction : $\Delta N_{X}=41 \pm 66$ (normalized to 34 days of data collection time).

Following the similar notations used by Ramberg-Snow and the VIP experiment papers, the number of possible PEP violating events, $\Delta N_{X}$, is related to the $\beta^{2} / 2$ parameter giving the probability of PEP violation [16] :

$$
\begin{aligned}
\Delta N_{X} & \geq \frac{1}{2} \beta^{2} N_{\text {new }} \frac{1}{10} N_{\text {int }} \times(\text { detection efficiency factor }) \\
& =\frac{\beta^{2}(\Sigma I \Delta t) D}{e \mu} \frac{1}{20} \times(\text { detection efficiency factor }) .
\end{aligned}
$$

Furthermore, the number of new electrons that pass through the conductor,

$$
N_{\text {new }}=(1 / e) \Sigma I \Delta t,
$$


is given by the electric charge $e$ of the electron, the intensity $I$ of the applied DC current, and the duration time $\Delta t$ of the measurement. The minimum number of internal scattering processes between a new electron and the atoms of the copper lattice, $N_{i n t}$, is of order $D / \mu$, where $D$ is the length of the copper strip $(10 \mathrm{~cm})$, and $\mu$ is the mean free path of electrons in copper. We follow the same assumption used in the VIP paper [17], that the capture probability of a new electron by an atom of the copper lattice is greater than $1 / 10$ of the scattering probability.

\section{Energy spectrum with 100 Ampere current}

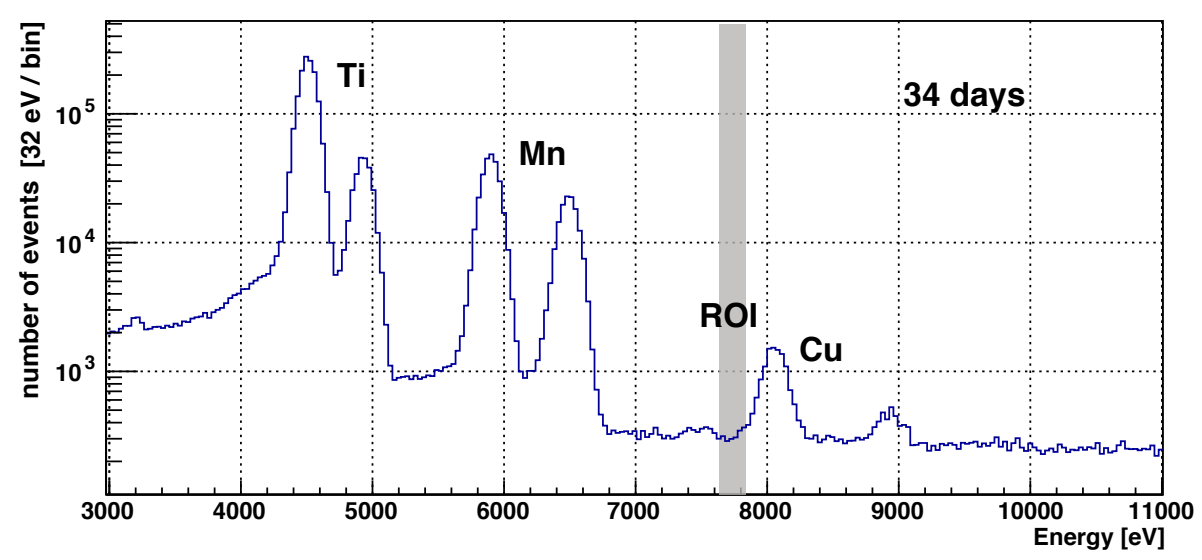

Energy spectrum without current

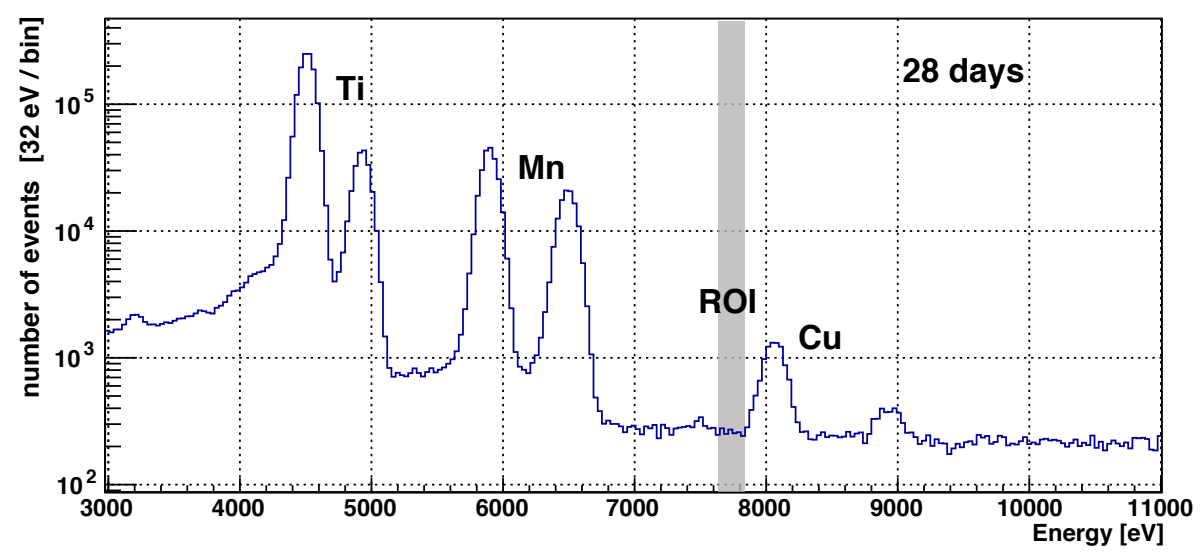

Figure 2. The energy spectra from all the SDDs, for data with and without applied DC current to the copper strip, taken during the physics run in late 2016 at the Laboratori Nazionali del Gran Sasso (LNGS).

The detection efficiency factor is evaluated with a Monte Carlo simulation based on Geant4.10 with realistic detector configuration, taking into account: the transmission rate of a copper $K_{\alpha} X$-ray that originates at a random position inside the copper strip and reaches the surface; the geometrical acceptance of the photons coming from the surface of the copper stip arriving at the six SDD detectors; the detection efficiency of a copper $K_{\alpha} X$-ray by the $450 \mu$ m-thick SDD unit, and the value is determined to be about $1 \%$.

With $D=10 \mathrm{~cm}, \mu=3.9 \times 10^{-6} \mathrm{~cm}, e=1.602 \times 10^{-19} \mathrm{C}, I=100 \mathrm{~A}$, and normalizing the measurement time with current to 34 days, using the three sigma upper bound of $\Delta N_{X}=41 \pm 66$ to give a $99.7 \%$ C.L., we get an upper limit for the $\beta^{2} / 2$ parameter:

$$
\frac{\beta^{2}}{2} \leq \frac{3 \times 66}{4.7 \times 10^{30}}=4.2 \times 10^{-29} .
$$




\section{Conclusions and Future Perspectives}

The first VIP-2 physics run from two months of data collection already gave a better limit than the VIP result obtained from three years of running.

In Figure 3, we show all the past experimental results of the PEP violation tests for electrons with a copper conductor, together with this work. The new result shows that in the planned data collection time of 3 to 4 years, the VIP-2 experiment can either set a new upper limit for the probability that the PEP is violated at the level of $10^{-31}$, improving the VIP experiment result by two orders of magnitude, or find the PEP violation, which would have profound implications in science and philosophy.

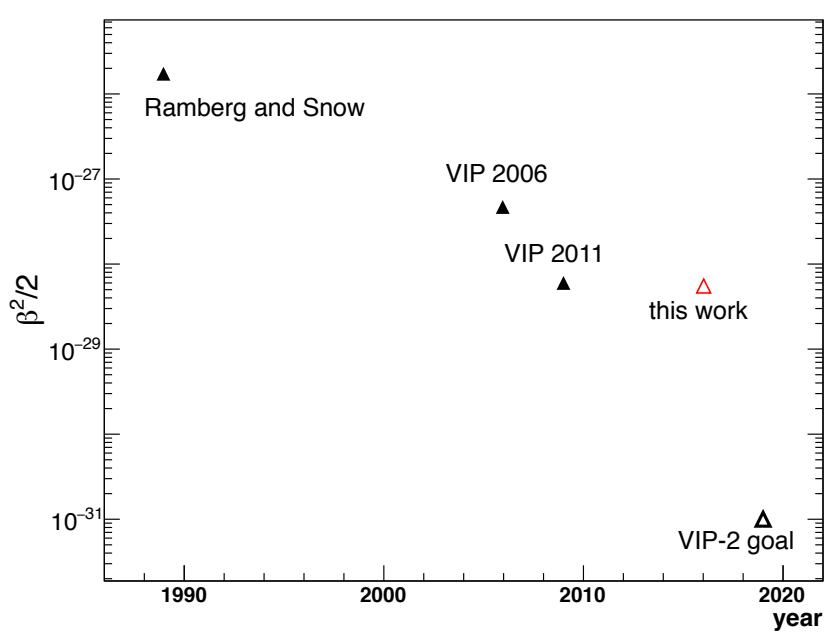

Figure 3. All the past results from Pauli exclusion principle (PEP) violation tests for electrons with a copper conductor, together with the result from this work and the anticipated goal of the VIP-2 experiment. Note that the result of this work comes from two months of data collection, and it is already compatible with the VIP result from three years of operation.

We conclude with the words of Lev Okun from his 1987 paper [18]: "The special place enjoyed by the Pauli principle in modern theoretical physics does not mean that this principle does not require further and exhaustive experimental tests. On the contrary, it is specifically the fundamental nature of the Pauli principle which would make such tests, over the entire periodic table, of special interest".

Acknowledgments: We thank H. Schneider, L. Stohwasser, and D. Stückler from Stefan-Meyer-Institut for their fundamental contribution in designing and building the VIP-2 setup. We acknowledge the very important assistance of the INFN-LNGS laboratory staff during all phases of preparation, installation and data taking. We thank the Austrian Science Foundation (FWF) which supports the VIP-2 project with the grant P25529-N20. We acknowledge the support from the EU COST Action CA15220, and from Centro Fermi ("Problemi aperti nella meccania quantistica" project). Furthermore, this paper was made possible through the support of a grant from the John Templeton Foundation (ID 58158). The opinions expressed in this publication are those of the authors and do not necessarily reflect the views of the John Templeton Foundation.

Author Contributions: Sergio Bertolucci, Catalina Curceanu, Jean-Pierre Egger, Carlo Guaraldo, Edoardo Milotti, Eberhard Widmann, Johann Zmeskal conceived and designed the experiment; Massimiliano Bazzi, Carolina Berucci, Alberto Clozza, Mihail Iliescu, Andreas Pichler, Hexi Shi, Florin Sirghi, Johann Zmeskal prepared the setup; Mihail Iliescu and Hexi Shi prepared the readout and data taking system; Andreas Pichler, Hexi Shi, Johann Zmeskal performed the detctor tests; Mario Bragadireanu, Alberto Clozza, Catalina Curceanu, Mihail Iliescu, Matthias Laubenstein, Johann Marton, Marco Miliucci, Andreas Pichler, Dorel Pietreanu, Kristian Piscicchia, Alessandro Scordo, Hexi Shi, Florin Sirghi, Johann Zmeskal contributed to the installation and data taking; Hexi Shi, Andreas Pichler, Michael Cargnelli, Luca De Paolis performed the data analysis; Sergio Bartalucci, Diana Laura Sirghi, Laura Sperandio, Oton Vazquez Doce provided details of the VIP analysis; Sergio Di Matteo provided theoretical support for the data analyses; Catalina Curceanu and Hexi Shi wrote the paper.

Conflicts of Interest: The authors declare no conflict of interest. 


$\begin{array}{ll}\text { Abbreviations } & \\ \text { PEP } & \text { Pauli Exclusion Principle } \\ \text { VIP(-2) experiment } & \text { VIolation of Pauli principle (-2) experiment } \\ \text { CCD } & \text { Carge Coupled Device } \\ \text { SDD } & \text { Silicon Drift Detector } \\ \text { NIM } & \text { Nuclear Instrumentation Module } \\ \text { VME } & \text { Versa Module Europa } \\ \text { QDC } & \text { Charge-to-Digital Converter } \\ \text { LNGS } & \text { Laboratori Nazionali del Gran Sasso } \\ \text { FWHM } & \text { Full Width Half Maximum } \\ \text { ROI } & \text { Region of Interest }\end{array}$

\section{References}

1. Messiah, A.M.L. Quantum Mechanics, Volume II; North-Holland: Amsterdam, The Netherlands, $1962 ;$ p. 595.

2. Messiah, A.M.L.; Greenberg, O.W. Symmetrization Postulate and Its Experimental Foundation. Phys. Rev. 1964, 136, B248.

3. Greenberg, O.W. Theories of Violation of Statistics. AIP Conf. Proc. 2000, 545, 113, doi: 10.1063/1.1337721.

4. Elliott, S.R.; LaRoque, B.H.; Gehman, V.M.; Kidd, M.F.; Chen, M. An Improved Limit on Pauli-Exclusion-Principle Forbidden Atomic Transitions. Found. Phys. 2012, 42, 1015-1030.

5. Ramberg, E.; Snow, G.A. Experimental Limit on a Small Violation of the Pauli Principle. Phys. Lett. B 1990, $238,438-441$.

6. Curceanu, C.; De Paolis, L.; Di Matteo, S.; Di Matteo, H.; Sperandio, S. Evaluation of the X-ray Transition Energies for the Pauli-Principle-Violating Atomic Transitions in Several Elements by Using the Dirac-Fock Method. Available online: http://www.lnf.infn.it/sis/preprint/detail.php?id=5330 (accessed on 23 June 2017).

7. Prange, R.; Girvin, S.M. The Quantum Hall Effect; Springer: New York, NY, USA, 1990.

8. Stern, A. Anyons and the quantum Hall effect-A pedagogical review. Ann. Phys. 2008, 323, $204-249$.

9. Curceanu, C.; Bartalucci, S.; Bertolucci, S.; Bragadireanu, M.; Cargnelli, M.; Di Matteo, S.; Egger, J.-P.; Guaraldo, C.; Iliescu, M.; Ishiwatari, T.; et al. Experiemntal tests of quantum mechanics-Pauli exclusion principle violation (the VIP experiment) and future perspective. J. Phys. Conf. Ser. 2011, 306, 012036, doi:10.1088/1742-6596/306/1/012036.

10. Bartalucci, S.; Bertolucci, S.; Bragadireanu, M.; Cargnelli, M.; Curceanu, C.; Di Matteo, S.; Egger, J.-P.; Guaraldo, C.; Iliescu, M.; Ishiwatari, T.; et al. The VIP experimental limit on the Pauli exclusion principle violation by electrons. Found. Phys. 2009, 40, 765-775.

11. Sperandio, L. New Experimental Limit on the Pauli Exclusion Principle Violation by Electrons From the VIP Experiment. Ph.D. Thesis, Tor Vergata University, Rome, Italy, 2008.

12. Shi, H.; Bartalucci, S.; Bertolucci, S.; Berucci, C.; Bragadireanu, A.M.; Cargnelli, M.; Clozza, A.; Curceanu, C.; De Paolis, L.; Di Matteo, S.; et al. Searches for the Violation of Pauli Exclusion Principle at LNGS in VIP(-2) experiment. J. Phys. Conf. Ser. 2016, 718, 042055, doi:10.1088/1742-6596/718/4/042055.

13. Pichler, A.; Bartalucci, S.; Bazzi, M.; Bertolucci, S.; Berucci, C.; Bragadireanu, M.; Cargnelli, M.; Clozza, A.; Curceanu, C.; De Paolis, L.; et al. Application of photon detectors in the VIP-2 experiment to test the Pauli Exclusion Principle. J. Phys. Conf. Ser. 2016, 718, 052030, doi:10.1088/1742-6596/718/5/052030.

14. Shi, H.; Bartalucci, S.; Bertolucci, S.; Berucci, C.; Bragadireanu, A.M.; Cargnelli, M.; Clozza, A.; Curceanu, C.; De Paolis, L.; Di Matteo, S.; et al. Testing the Pauli Exclusion Principle for electronics at LNGS. Phys. Procedia 2015, 62, 522-559.

15. Marton, J.; Bartalucci, S.; Bertolucci, S.; Berucci, C.; Bragadireanu, M.; Cargnelli, M.; Curceanu, C.; Di Matteo, S.; Egger, J.-P.; Guaraldo, C.; et al. Testing the Pauli Exclusion Principle for Electrons. J. Phys. Conf. Ser. 2013, 447, 012060, doi:10.1088/1742-6596/335/1/012060.

16. Greenberg, O.W.; Mohapatra, R.N. Local Quantum Field Theory of Possible Violation of the Pauli Principle. Phys. Lett. 1987, 59, 2507. 
17. VIP Collaboration; Bartalucci, S.; Bertolucci, S.; Bragadireanu, M.; Cargnelli, M.; Catitti, M.; Curceanu, C.; Di Matteo, S.; Egger, J.-P.; Guaraldo, C.; et al. New experimental limit on the Pauli exclusion principle violation by electrons. Phys. Lett. B 2006, 641, 18-22.

18. Okun, L. Possible violation of the Pauli principle in atoms. JETP Lett. 1987, 46, 529-532.

(C) 2017 by the authors. Licensee MDPI, Basel, Switzerland. This article is an open access article distributed under the terms and conditions of the Creative Commons Attribution (CC BY) license (http:/ / creativecommons.org/licenses/by/4.0/). 